\title{
Robot-assisted stereotactic biopsy of pediatric brainstem and thalamic lesions
}

\author{
Mihir Gupta, MD, ${ }^{1}$ Tiffany M. Chan, MD, ${ }^{2}$ David R. Santiago-Dieppa, MD, ${ }^{1}$ Anudeep Yekula, MBBS, ${ }^{3}$ \\ Carlos E. Sanchez, MD, ${ }^{4}$ Jennifer D. Elster, MD, ${ }^{5}$ John R. Crawford, MD, MS, ${ }^{5}$ \\ Michael L. Levy, MD, PhD, ${ }^{1,6}$ and David D. Gonda, MD ${ }^{1,6}$
}

\begin{abstract}
${ }^{1}$ Department of Neurosurgery, University of California, San Diego, La Jolla, California; ${ }^{2}$ Michael E. DeBakey Department of Surgery, Baylor College of Medicine, Houston, Texas; ${ }^{3}$ Department of Neurosurgery, Massachusetts General Hospital, Boston, Massachusetts; ${ }^{4}$ Department of Neurosurgery, Children's National Health System, Washington, DC; and ${ }^{5}$ Department of Neurology and ${ }^{6}$ Division of Neurosurgery, Rady Children's Hospital, San Diego, California
\end{abstract}

\begin{abstract}
OBJECTIVE Biopsies of tumors located in deep midline structures require highly accurate stereotaxy to safely obtain lesional tissue suitable for molecular and histological analysis. Versatile platforms are needed to meet a broad range of technical requirements and surgeon preferences. The authors present their institutional experience with the robotic stereotactic assistance (ROSA) system in a series of robot-assisted biopsies of pediatric brainstem and thalamic tumors.
\end{abstract}

METHODS A retrospective analysis was performed of 22 consecutive patients who underwent 23 stereotactic biopsies of brainstem or thalamic lesions using the ROSA platform at Rady Children's Hospital in San Diego between December 2015 and January 2020.

RESULTS The ROSA platform enabled rapid acquisition of lesional tissue across various combinations of approaches, registration techniques, and positioning. No permanent deficits, major adverse outcomes, or deaths were encountered. One patient experienced temporary cranial neuropathy, and 3 developed small asymptomatic hematomas. The diagnostic success rate of the ROSA system was $91.3 \%$.

CONCLUSIONS Robot-assisted stereotactic biopsy of these lesions may be safely performed using the ROSA platform. This experience comprises the largest clinical series to date dedicated to robot-assisted biopsies of brainstem and diencephalic tumors.

https://thejns.org/doi/abs/10.3171/2020.7.PEDS20373

KEYWORDS robotic surgery; brainstem; thalamus; pediatric brain tumor; ROSA robot; midline glioma; oncology

$\mathrm{T}$ HE modern classification of tumors of the CNS combines genetic and histological signatures to formulate clinically relevant integrated diagnoses. ${ }^{1}$ Lesions such as diffuse intrinsic pontine gliomas (DIPGs) that were previously diagnosed and treated based on radiographic findings alone may now require biopsy to obtain accurate diagnoses and determine clinical trial eligibility. ${ }^{2,3}$ Neurosurgeons are thus required to offer safe, minimally invasive, and cost-effective solutions to obtain tissue suitable for molecular analysis.

Lesions in eloquent locations such as the brainstem and thalamus are frequently difficult to access via open surgical approaches and require highly accurate stereotactic tools to biopsy safely. Frame-based stereotaxy has historically been the gold standard for successfully performing these proce- dures. Recent studies have explored frameless procedures and new technologies such as robotic assistance to improve surgical workflow, reduce operative time, and perform other procedures such as endoscopic third ventriculostomies (ETVs) during the same operation as biopsies.

Robotic assistance has been described in several large clinical series of brain biopsy procedures, predominantly for supratentorial pathologies. ${ }^{3-10}$ Emerging evidence in smaller series suggests that robot-assisted biopsies may also yield good outcomes in brainstem lesions. ${ }^{11-14} \mathrm{We}$ describe our institutional series of pediatric brainstem and thalamic tumor biopsies using the robotic stereotactic assistance (ROSA) platform and discuss the results in the context of the growing literature on robot-assisted biopsies of these challenging lesions.

ABBREVIATIONS DIPG = diffuse intrinsic pontine glioma; ETV = endoscopic third ventriculostomy; EVD = external ventricular drain; FFPE = formalin-fixed paraffin-embedded; $\mathrm{H3}=$ histone 3; IQR = interquartile range; LGG = low-grade glioma; ROSA = robotic stereotactic assistance; VP = ventriculoperitoneal.

SUBMITTED May 19, 2020. ACCEPTED July 20, 2020.

INCLUDE WHEN CITING Published online December 25, 2020; DOI: 10.3171/2020.7.PEDS20373. 


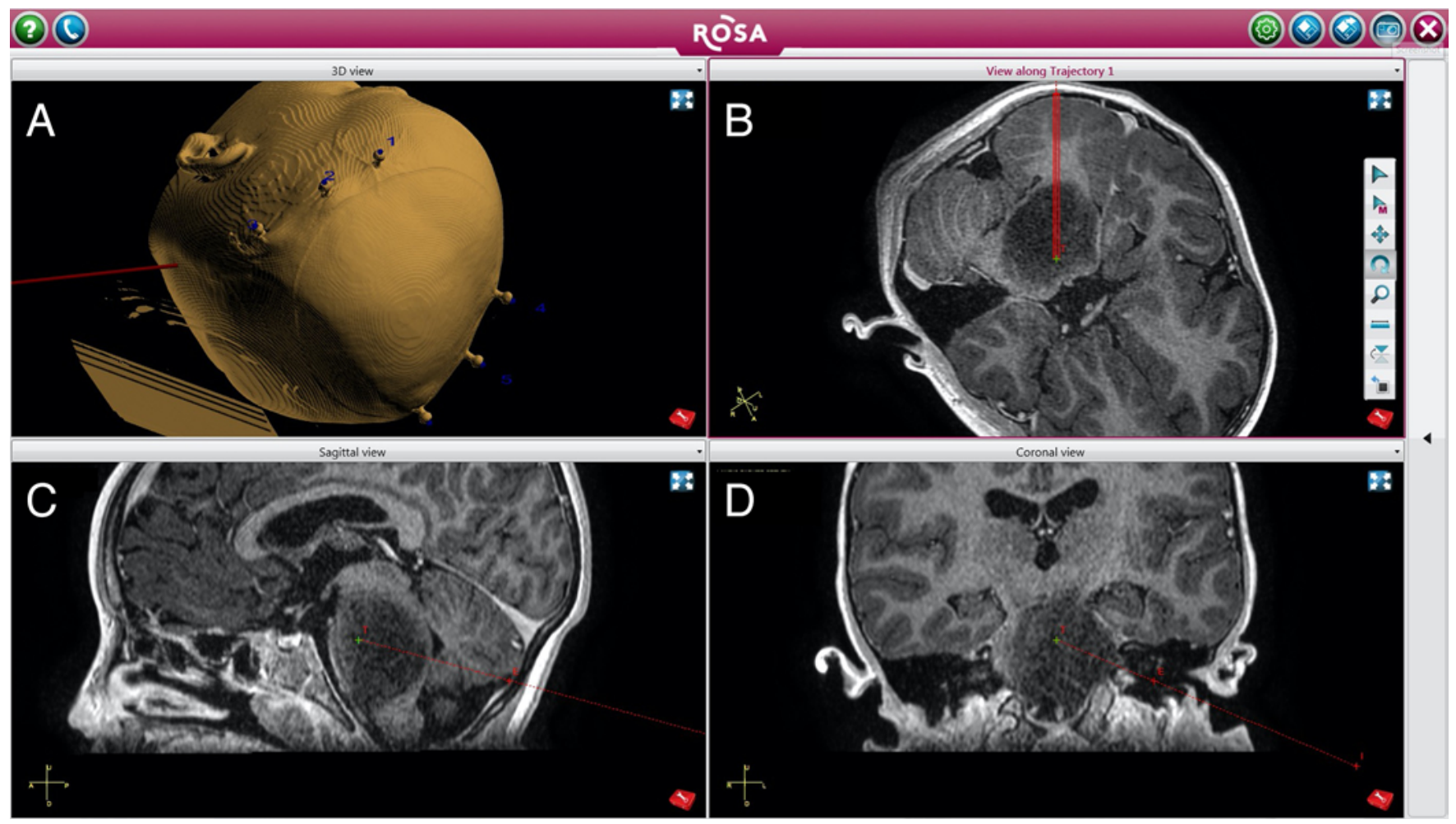

FIG. 1. Surgical planning for robot-assisted biopsy of a pontine lesion using the Rosana software. Panel $A$ shows frameless surface reconstruction with in-bone fiducial markers in place. The remaining panels (B-D) are axial, sagittal, and coronal, respectively, to the planned biopsy trajectory (red line). Figure is available in color online only.

\section{Methods \\ Patient Population}

We retrospectively evaluated 22 consecutive patients who underwent 23 robot-assisted biopsies of brainstem or thalamic lesions between December 2015 and January 2020 at Rady Children's Hospital in San Diego. The study population included 9 male and 13 female patients ranging from 3 to 24 years of age at presentation (median age 9.1 years, interquartile range [IQR] 7.3-11.2 years). IRB approval was obtained for this retrospective study.

\section{Preoperative Planning}

Preoperatively, all patients underwent high-resolution, gadolinium-enhanced 1.5-T MRI of the brain (Discovery MR450, GE Healthcare). T1-, T2-, diffusion-, and susceptibility-weighted as well as FLAIR sequences were performed in all cases. Postcontrast studies included 3D T1-weighted fast-spoiled gradient recalled sequences with fat suppression. Additionally, a noncontrast high-resolution CT scan with continuous $0.62-\mathrm{mm}$ slice thickness was obtained preoperatively in 9 cases and intraoperatively in 7 cases; in the remaining 7 cases, navigation was performed with MRI alone. CT and MR images were loaded into the manufacturer-provided planning software (Rosana, Medtech). Images were coregistered using a rigid and linear algorithm, and manually corrected by the surgeon based on anatomical landmarks. Trajectories and targets were selected as previously described. ${ }^{6}$ The most avidly enhancing regions of lesions were selected for biopsy targets. Occasionally, the enhancing region was limited to a very small nodule at the distal portion of the lesion (Fig. 1).

\section{Surgical Procedure}

The ROSA robotic platform (Medtech) was used in all procedures. Following induction of general anesthesia and standard endotracheal intubation, invasive bone fiducials were inserted in 5 cases. A Leksell stereotactic frame was applied in 1 case, while the remainder were conducted with frameless surface registration. Intraoperative CT scans were performed with patients under anesthesia, immediately prior to positioning. For frameless navigation, the head was secured in a Sugita frame (Mizuho, Inc.), which was attached to the robotic device during patient positioning (Fig. 2). The robot's automatic contactless surface registration capability was used to capture points of the face and scalp, with the patient in the operative position. Share-controlled manual and robotic registration was utilized in cases with fiducial markers or a stereotactic frame. Registration accuracy was confirmed by the surgeon on external anatomical landmarks. Patients were then draped and the robotic arm was automatically aligned to the previously planned trajectory. In all cases in which an ETV was also planned, the biopsy was performed first to avoid introducing anatomical shifts that might affect accuracy of the stereotactic biopsy.

A small stab incision was made with a No. 15 scal- 


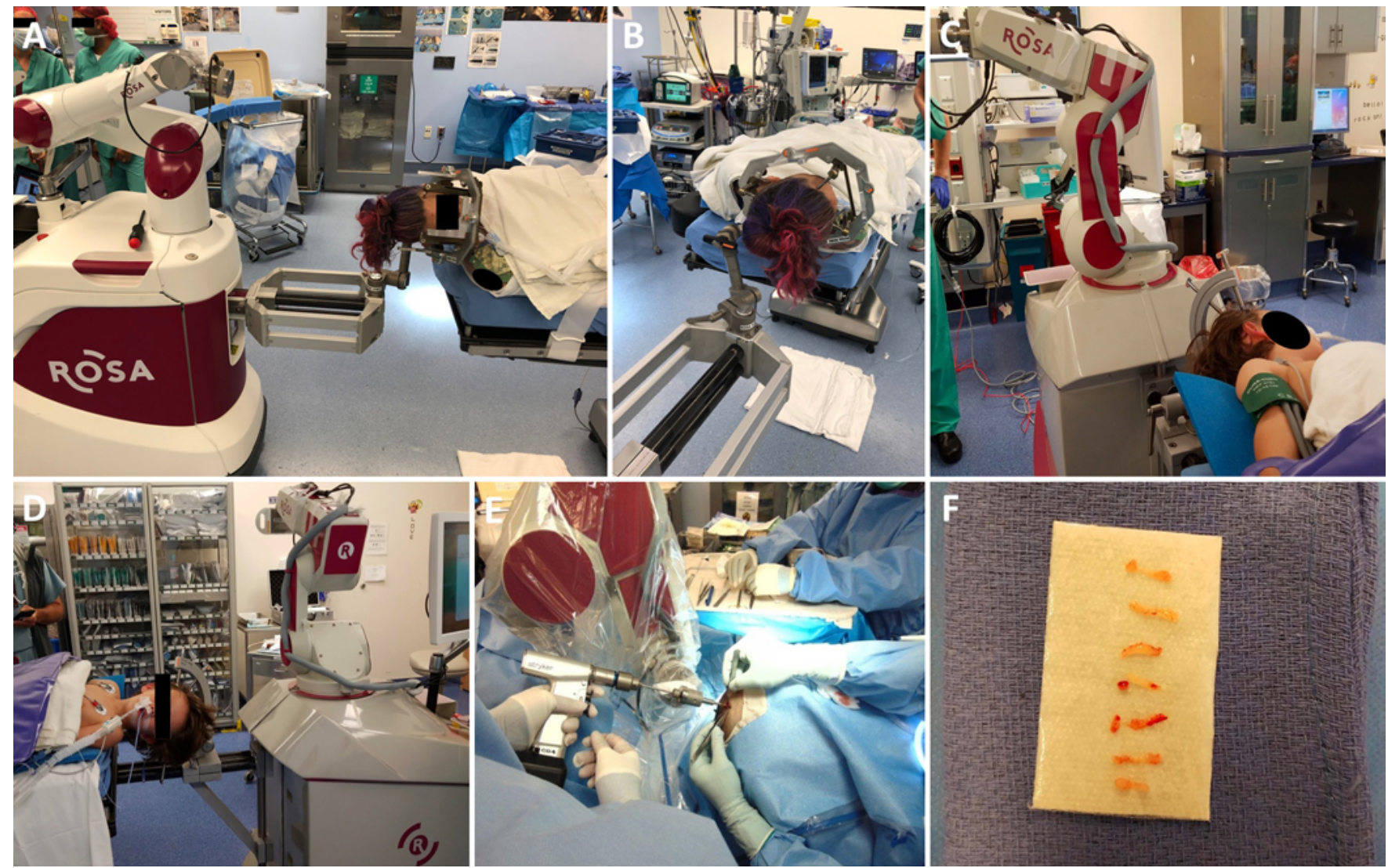

FIG. 2. Stereotactic biopsy of brainstem lesions using the ROSA system. A-D: Both stereotactic frames (A and B) or headrests with pins ( $C$ and $D)$ may be affixed to the robot arm adapter. $E$ : The robotic arm automatically moves into position for the planned biopsy trajectory. The arm holds a reducer to guide the drill and biopsy needle. F: Multiple core biopsies are taken from each site. Figure is available in color online only.

pel at the site indicated by the robotic laser guide. A 2.4-mm hole was made in the skull using a high-speed drill guided by the steel bushing of the robotic arm. The dura was opened with a K-wire. The steel bushing was replaced with polyetheretherketone bushing sized for the biopsy needle. A 1.8-mm diameter Varioguide (Brainlab, Inc.) stereotactic biopsy needle with a $10-\mathrm{mm}$ side-cut specimen window was passed through the robot arm to the desired target. Core biopsies of lesional tissue were then acquired by standard negative-pressure technique. The needle was sequentially rotated to capture 4 separate specimens at each of the desired sites. For larger lesions, the biopsy needle was withdrawn about $5 \mathrm{~mm}$ and an additional set of quadrantal biopsies was obtained at the new depth. The biopsy needle was then withdrawn completely from the head and the wound was closed with a single 4-0 absorbable monofilament suture.

In cases involving ETV, the robotic arm was repositioned to Kocher's point and a new burr hole was made. ${ }^{15}$ A rigid $0^{\circ}$ endoscope was introduced through the robot arm into the ventricles following the preplanned trajectory. A Bugbee wire (Medline) was used to perform a ventriculostomy, which was then dilated with a 3-Fr Fogarty balloon. The wound was closed and the robotic arm repositioned to the site of the stereotactic biopsy.
Acquisition of lesional tissue was confirmed by intraoperative histopathologic analysis. Formalin-fixed paraffinembedded (FFPE) sections were prepared and analyzed by standard histopathologic and immunohistochemical analysis for final diagnosis. When clinically indicated by consensus among the neurosurgical, neuropathology, and neurooncology teams, selected FFPE specimens were also submitted for next-generation sequencing and microarray analysis; assays were performed by our clinical laboratory or the facilities designated by clinical trial protocols.

\section{Results}

Clinical characteristics of the cohort are summarized in Table 1. Seventeen biopsies (74\%) were performed for newly diagnosed lesions, and 6 (26\%) for progressive lesions. Clinical presentation included cranial neuropathies, pyramidal tract dysfunction, cerebellar signs, and symptoms of elevated intracranial pressure. In the majority of cases $(65 \%)$ no prior treatment had been performed. Six patients $(26 \%)$ had previously received CSF diversion by ventriculoperitoneal (VP) shunting (3 patients), ETV (2 patients), or external ventricular drain (EVD; 1 patient).

Lesions predominantly arose from the pons, with variable degrees of involvement of other brainstem, posterior 
TABLE 1. Clinical characteristics of patients undergoing robotassisted biopsy

\begin{tabular}{|c|c|}
\hline Characteristic & Value \\
\hline Total no. of biopsies performed & 23 \\
\hline Total no. of patients & 22 \\
\hline Females, n (\%) & $13(59)$ \\
\hline Median age at surgery (IQR), yrs & $9.1(7.3-11.2)$ \\
\hline \multicolumn{2}{|l|}{ Stage at biopsy, n (\%) } \\
\hline New lesion & $17(74)$ \\
\hline Progression & $6(26)$ \\
\hline \multicolumn{2}{|l|}{ Symptoms at presentation, $\mathrm{n}(\%)$} \\
\hline Ataxia & $13(57)$ \\
\hline Headache & $7(30)$ \\
\hline Emesis & $7(30)$ \\
\hline Lethargy & $2(9)$ \\
\hline Hemiparesis & $8(35)$ \\
\hline Cranial neuropathies & $20(87)$ \\
\hline Nystagmus & $3(13)$ \\
\hline Radiographic progression alone & $2(9)$ \\
\hline Median symptom duration (IQR), mos & $0.9(0.5-2)$ \\
\hline \multicolumn{2}{|l|}{ Prior treatment, $\mathrm{n}(\%)$} \\
\hline None & $15(65)$ \\
\hline VP shunt & $3(13)$ \\
\hline ETV & $2(9)$ \\
\hline EVD & $1(4)$ \\
\hline Biopsy & $2(9)$ \\
\hline Radiation & $4(17)$ \\
\hline Intratumoral chemotherapy & $2(9)$ \\
\hline Peptide vaccine & $1(4)$ \\
\hline
\end{tabular}

fossa, and diencephalic structures. Patient-level anatomical characteristics and operative data are shown chronologically in Fig. 3 and summarized in Supplemental Table 3 . Biopsy targets were located in the pons (16 cases), tectum (3 cases), medulla ( 2 cases), or thalamus ( 2 cases). Patients were positioned supine with their face looking upward for tectal and thalamic biopsies. Positioning was supine with head turned $45^{\circ}$ when performing a transcerebellar approach to pontine or medulla structures. This permitted concurrent performance of ETV during the same procedure (7 patients) without repositioning when needed.

Operative duration was a function of the procedures performed (e.g., biopsy alone vs biopsy with ETV), intraoperative CT scan, and learning curve, as we gained familiarity and comfort with the ROSA system (Supplemental Fig. 1). In cases in which intraoperative CT was not performed, the median surgical time was 21 minutes for biopsy alone and 64 minutes for biopsy and ETV.

Postoperative outcomes are described in Table 2. Three patients developed asymptomatic intracranial hematomas following biopsy, including 2 intratumoral subcentimeter hemorrhages and 1 extraaxial hematoma less than $5 \mathrm{~mm}$ in diameter. Patients were discharged on the first postoperative day in 11 cases $(47.8 \%)$ and on the second post- operative day in 4 cases (17.4\%). One patient experienced transient new swallowing difficulties, which resolved after 2-3 weeks. There were no permanent deficits, infectious sequelae, major adverse outcomes, or deaths related to the biopsy procedures. In 18 patients for whom survival data were available, the median overall survival was 13.5 months (range 2-61 months); at the time of analysis, 7 patients were deceased and 11 alive.

Diagnostic and molecular genetic data are shown in Table 3. Biopsy specimens were a median volume of 0.07 $\mathrm{cm}^{3}$ and yielded diagnostic tissue in 21 cases (91.3\%). The majority of cases were high-grade glioma $(n=14,64 \%)$, including 9 cases of histone 3 (H3) K27M-mutant diffuse midline glioma. Next-generation sequencing was performed in the majority of cases and revealed mutations in a wide array of canonical cancer-related genes. Pathologic and molecular diagnosis determined subsequent steps in treatment, including radiosurgery, chemotherapy, epigenetic therapy, craniotomy, and clinical trial enrollment.

Diagnostic tissue was not obtained in 2 cases, both of which were located in the pons and medulla and were ultimately diagnosed as low-grade gliomas (LGGs). In the first case, the tumor was impenetrable by the biopsy needle; definitive diagnosis of a neurofibromatosis type 1-associated brainstem glioma was established by craniotomy and subtotal resection. In the second case, the patient responded to empirical treatment for LGG, confirming the presumed diagnosis. Postbiopsy imaging showed that the trajectories in both instances were accurate until the needle reached the medulla, where it appears that the medulla was not sufficiently fixed in position to hold the course of the needle.

\section{Discussion}

The first robot-assisted stereotactic biopsy of a brain lesion was performed collaboratively between neurosurgeons and electrical engineers in 1985, using a modified General Motors PUMA 200-series industrial robot in conjunction with a stereotactic frame. ${ }^{16}$ Drake and colleagues subsequently utilized the same system to obtain biopsies of deep benign astrocytomas in pediatric patients. ${ }^{17} \mathrm{Al}-$ though these studies offered early proof of principle regarding the feasibility and safety of robot assistance in navigating and stabilizing instruments for neurosurgical procedures, it was not until the advent of MRI-guided stereotaxy that robotic systems gained widespread use in cranial neurosurgery. Improvements in accuracy, safety, and user-friendly modalities such as frameless surface registration have led to the incorporation of robotic assistance into procedures including biopsy, neuroendoscopy, depth electrode implantation, neuromodulation treatment, and lesion ablation. ${ }^{4}$

In a meta-analysis of 735 stereotactic biopsies of pediatric brainstem tumors across 18 published studies, Hamisch and colleagues found a $96.1 \%$ diagnostic success rate, with $0.6 \%$ mortality and $6.7 \%$ morbidity (including $0.6 \%$ permanent morbidity).$^{18}$ The meta-analysis included 17 series without robot assistance and 1 early series of 33 robot-assisted procedures. ${ }^{5}$ To our knowledge, a total of 104 robot-assisted biopsies of brainstem lesions have been 
Patient ID

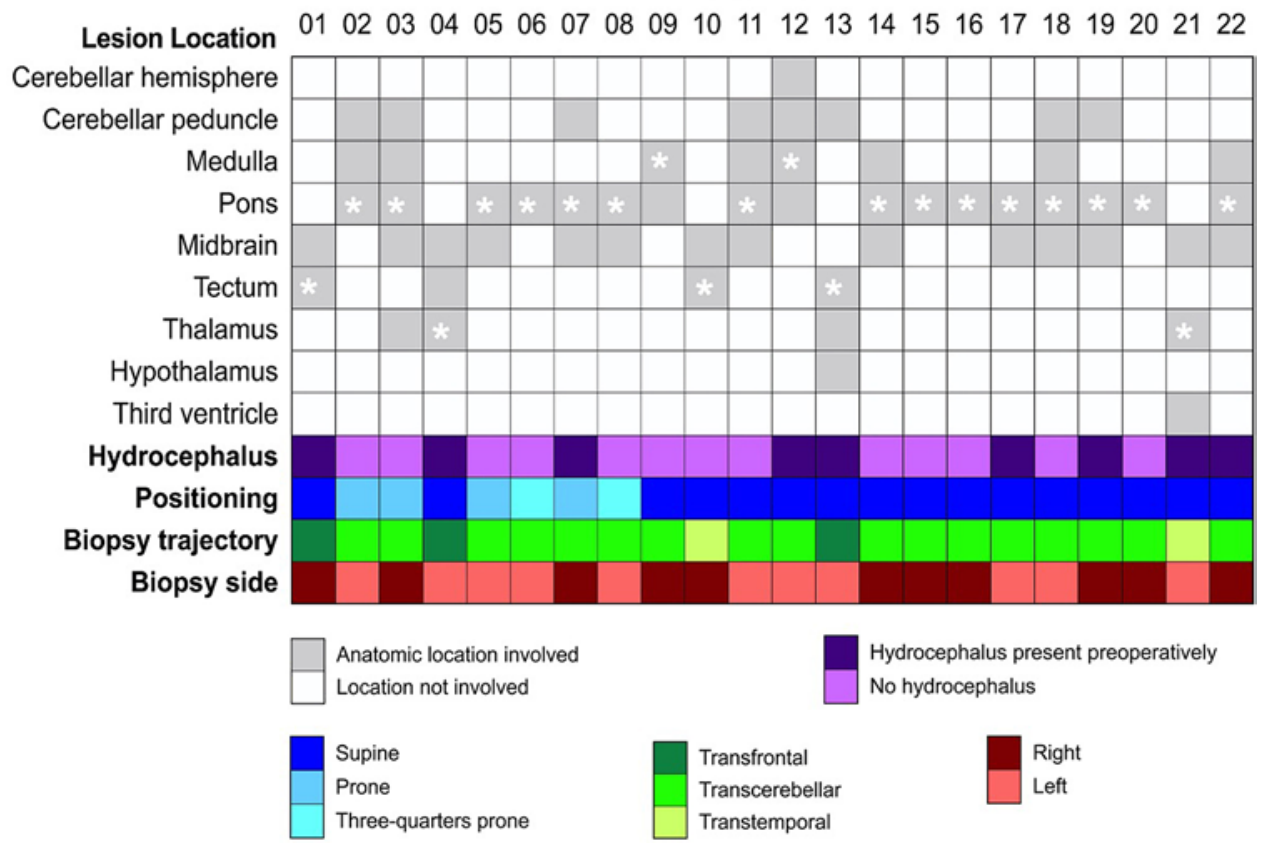

FIG. 3. Anatomical characteristics and operative strategies in patients undergoing robot-assisted stereotactic biopsy of brainstem and diencephalic lesions. Individual patient-level data are shown. Patients are listed in chronological order of procedures. Asterisks indicate the region sampled during biopsy. Patient 7 underwent a second biopsy by the same approach following lesion recurrence (not displayed). Figure is available in color online only.

described across adult and pediatric populations. This includes 41 cases in series dedicated to brainstem biopsies alone (Supplemental Table 1) $)^{11-14}$ and 63 cases included in larger institutional series of various robot-assisted neurosurgical procedures (Supplemental Table 2). ${ }^{3-10}$ Collectively, the diagnostic success rate for robot-assisted brainstem biopsies was $94.7 \%$ (54/57 cases with available data), with $9.8 \%$ temporary morbidity (5/51 cases), $3.9 \%$ permanent morbidity (2/51 cases), and no mortality (0/51 cases).

The relatively few published series of robot-assisted brainstem biopsy procedures to date are highly variable with respect to factors such as patient positioning, robotic system, use of stereotactic frames, choice of headrest, biopsy trajectory, target selection, use of intraoperative CT scan, and stereotactic registration method. Although randomized comparisons are lacking, retrospective analyses across multiple brainstem biopsy series have demonstrated similar outcomes from transfrontal and transcerebellar approaches, ${ }^{5,11,19}$ as well as frameless versus frame-based stereotaxy. ${ }^{20,21}$ Whereas transcerebellar trajectories have traditionally been performed in the prone position, 6,7 emerging evidence including our experience suggests that these approaches may be equally feasible in the supine position. ${ }^{11-14}$ Supine positioning may also reduce positioning time, permit simultaneous performance of procedures such as ETV, and enable frameless facial surface matching registration.

In our practice, frameless facial surface matching registration to MRI alone may be used in cases of larger lesions. We found that preoperative CT scans fused to MRI could improve facial surface matching for targets smaller than approximately $1 \mathrm{~cm}$ in diameter. We performed intraoperative CT scans only in cases when in-bone fiducials were used, namely for small targets located in deep structures such as the tectum. In 1 early case we used a Leksell frame instead of in-bone fiducials; although this allowed us to forgo placing invasive fiducials, we subsequently preferred to use fiducials due to comparable accuracy and expedited workflow. Future studies in larger cohorts are needed to build upon these observations and formulate practice guidelines.

Our experience corroborates previous reports that the ROSA system is a user-friendly, versatile platform that may be adapted to surgeon preferences and an array of technical requirements with a favorable safety profile and short learning curve. . $^{4,6,8,11,12}$ In comparison to standard stereotactic techniques, we have found robotic assistance to enable greater versatility and speed in patient positioning. For transcerebellar approaches, the rigid drill guide of the robot prevents bending or misalignment of the drill bit on the oblique surface of the occipital bone. Whereas the base or arc of stereotactic frames may occasionally obstruct approaches to the posterior fossa, this obstacle is not encountered with frameless stereotactic biopsies. Furthermore, when additional biopsy passes along the same trajectory need to be performed, the robotic system can be directed to advance or retract the catheter by the desired length. Finally, our learning curve, displayed chronologically at the individual patient level in Supplemental Fig. 1, highlights the rapid operative times that can be achieved with robotic assistance.

The increased use of intraoperative stereotactic and ro- 
TABLE 2. Outcomes following robot-assisted stereotactic biopsy

\begin{tabular}{lc}
\hline \multicolumn{1}{c}{ Characteristic } & Value \\
\hline Median length of stay (IQR), days & $2(1-3)$ \\
\hline Postop hematoma, $\mathrm{n}(\%)$ & $20(87)$ \\
\hline None & $2(9)$ \\
\hline Intralesional & $1(4)$ \\
\hline Extraaxial & $22(96)$ \\
\hline New postop deficit, $\mathrm{n}(\%)$ & $1(4)$ \\
\hline None & \\
\hline Temporary swallowing difficulty & $3(13)$ \\
\hline Additional surgery after biopsy, $\mathrm{n}(\%)$ & $4(17)$ \\
\hline Craniotomy & $5(22)$ \\
\hline VP shunt & $1(4)$ \\
\hline ETV & $8(35)$ \\
\hline Repeat stereotactic biopsy & $12(52)$ \\
\hline Other subsequent treatment, $\mathrm{n}(\%)$ & $4(17)$ \\
\hline Radiation & $1(4)$ \\
\hline Chemotherapy & $1(4)$ \\
\hline HDAC inhibitor & $9(3-17)$ \\
\hline Peptide vaccine & \\
\hline Intratumoral treatment & \\
\hline Median follow-up (IQR), mos & \\
\hline HDAC $=$ histone deacetylase. & \\
\hline
\end{tabular}

botic modalities has coincided with a paradigm shift in the classification of CNS tumors. In 2016, the WHO formulated an integrated molecular and histological diagnostic framework for many CNS neoplasms, a major departure from the previous classification system. ${ }^{22}$ The updated molecular classifications have direct implications for prognosis, therapeutic options, and clinical trial eligibility. Additionally, even small amounts of tissue specimens used in research studies have enabled groundbreaking translational insights. Tissue may also be needed to accurately distinguish posttreatment effects such as radiation necrosis and pseudoprogression from true progression, each of which has different diagnostic and therapeutic implications. The need for accurate molecular diagnosis has, in turn, driven demand for acquisition of diagnostic tissue, including from anatomically unresectable tumors and suspected recurrences of previously treated lesions.

In this context, neurosurgeons may be among the first specialists to interface with patients following the discovery of a new or recurrent brain lesion. Interdisciplinary collaboration with neurooncology and neuropathology providers is critical to conducting meaningful informed discussions with families prior to contemplating surgical intervention. It is also incumbent upon the surgeon to formulate strategies for safe, minimally invasive biopsy performance, with attention to proper handling of specimens for molecular profiling. Safety and accuracy are critical for pediatric patients in whom developing structures may be particularly vulnerable to injury. ${ }^{23}$

These considerations are also relevant to deep-seated lesions of the brainstem and diencephalon. The majority of our cohort consists of pontine tumors, previously known
TABLE 3. Specimen volume, pathologic diagnosis, and molecular genetic alterations

\begin{tabular}{lc}
\hline \multicolumn{1}{c}{ Characteristic } & Value \\
\hline Median tissue volume (IQR), $\mathrm{cm}^{3}$ & $0.07(0.05-0.12)$ \\
\hline Next-generation sequencing performed, $\mathrm{n}(\%)$ & $12(55)$ \\
\hline Diagnosis, $\mathrm{n}(\%)$ & $2(9)$ \\
\hline Pilocytic astrocytoma & $2(9)$ \\
\hline Diffuse astrocytoma, WHO grade II & $3(14)$ \\
\hline Other LGG* & $9(41)$ \\
\hline Diffuse midline glioma, H3 K27M-mutant & $3(14)$ \\
\hline Glioblastoma & $2(9)$ \\
\hline Other high-grade glioma† & $1(5)$ \\
\hline Radiation necrosis & \\
\hline Coding region mutations or copy number & \\
alterations detected, $\mathrm{n}(\%)$ & $2(9)$ \\
\hline ARID2 & $2(9)$ \\
\hline CDKN2A & $2(9)$ \\
\hline CDKN2B & $2(9)$ \\
\hline FGFR1 & $9(41)$ \\
\hline H3F3A & $3(14)$ \\
\hline KRAS & $1(5)$ \\
\hline NF1 & $1(5)$ \\
\hline NRAS & $2(9)$ \\
\hline PDGFRA & $4(18)$ \\
\hline TP53 & $2(9)$ \\
\hline TSC1 & $1(5)$ \\
\hline KIAA1549-BRAF fusion detected, $\mathrm{n}(\%)$ & \\
\hline
\end{tabular}

* Includes 1 case each of neurofibromatosis type 1-associated low-grade brainstem glioma, tectal LGG with KRAS mutation, and empirically diagnosed LGG.

† Includes 1 case each of WHO grade III and WHO grade III-IV glioma.

categorically as DIPGs. These lesions comprise $10 \%$ of pediatric brain tumors ${ }^{24}$ and are the leading cause of brain tumor-related mortality in children. ${ }^{25}$ Until recently, a diagnosis was made using radiographic findings alone, and neurosurgical intervention was indicated only to treat obstructive hydrocephalus. ${ }^{26}$ Later, a series of seminal studies revealed that nearly $80 \%$ of DIPG cases harbor the H3 K27M mutation. ${ }^{27}$ The mutation is now considered pathognomonic for the new entity of H3 K27M-mutant diffuse midline glioma, which carries a WHO grade IV designation due to universally poor prognosis irrespective of histological features. ${ }^{22}$ Lesional tissue is required for detection of the H3 K27M mutation, which may be performed by immunohistochemistry or direct genetic sequencing. Although many studies have demonstrated that biopsy of brainstem lesions in pediatric patients is safe and can guide molecularly targeted therapies, ${ }^{2,3,28,29}$ it is important to note that these procedures are not yet universally considered standard of care and may be limited to large centers.

In light of the critical role of tissue diagnosis, some authors now advocate that stereotactic brainstem biopsy be considered in all cases of newly diagnosed DIPG. ${ }^{3}$ The fre- 
quently poor medical condition and guarded prognosis in this patient population also highlight the need to minimize time under anesthesia, surgery-related risks, and length of hospital stay. These lesions must also be accurately distinguished from low- and high-grade $\mathrm{H} 3$ wild-type pontine gliomas, which represented 3 and 4 cases, respectively, of new lesions in our cohort involving the pons.

In this context, the 2 pontomedullary LGGs in our cohort that yielded nondiagnostic biopsies are also instructive. Biopsy of the medullary portion of the lesion was attempted in both cases because this was the region of most avid contrast enhancement. Both cases were performed using frameless stereotaxy; preoperative CT was merged to the MRI for 1 case, while the other was registered to the MRI alone. Postoperative imaging confirmed the correct trajectory of the approach. Thus, we concluded that the mobility of the anatomical structure likely prevented adequate needle penetration and stability. Because adjuncts such as intraoperative CT would not mitigate these factors, we propose that the pontine portion of lesions may be a preferable stereotactic biopsy target, whereas medullary lesions may require open biopsies. This technical consideration has not been studied in the setting of robot-assisted brainstem biopsies (Supplemental Tables 1 and 2) but has direct implications for target selection in suspected brainstem glioma biopsy planning.

Similar to prior reports, the scope of this study is limited to evaluating the feasibility, safety, and operative considerations of robot-assisted biopsy procedures. Future investigations may build upon these observations to analyze factors such as cost-benefit analysis of robot-assisted procedures. These studies will require larger cohorts and careful selection of comparable procedures to control for factors such as choice of stereotactic navigation, use of CT scans, disease subtypes, and postoperative care plans. The cost-benefit of the initial investment in robotic technology must also account for the utility of the platform for performing a wide range of neurosurgical procedures.

This study is subject to the standard limitations of retrospective analysis in heterogeneous patient cohorts of similar size. Future studies are warranted to compare the ROSA platform with other robotic systems. Best practices regarding stereotactic registration, imaging protocols, and operative workflows must also be defined. Innovations such as stable bone contact between the robotic arm and the skull pioneered in other robotic systems ${ }^{7}$ may also be applied to the ROSA device to improve accuracy and stability. Opportunities to reduce bleeding risk, such as improved trajectories or needle design, should also be explored. Finally, the possibilities of integrating robotic technology with modalities such as convention-enhanced therapeutic delivery, ${ }^{30}$ liquid biopsy, ${ }^{31}$ and intraoperative diagnostics ${ }^{32}$ present exciting innovative frontiers.

\section{Conclusions}

We present the largest clinical series to date of robot-assisted biopsies specifically of brainstem and diencephalic lesions, as well as the largest series to utilize the ROSA platform for biopsies of brainstem lesions. Our experience supports the use of the ROSA system for stereotactic guid- ance and instrument stabilization to rapidly and safely acquire diagnostic tissue from deep, anatomically unresectable lesions in pediatric patients.

\section{References}

1. Brat DJ, Aldape K, Colman H, et al. cIMPACT-NOW update 5: recommended grading criteria and terminologies for IDHmutant astrocytomas. Acta Neuropathol. 2020;139(3):603-608.

2. Lin GL, Wilson KM, Ceribelli M, et al. Therapeutic strategies for diffuse midline glioma from high-throughput combination drug screening. Sci Transl Med. 2019;11(519): eaaw0064.

3. Williams JR, Young CC, Vitanza NA, et al. Progress in diffuse intrinsic pontine glioma: advocating for stereotactic biopsy in the standard of care. Neurosurg Focus. 2020;48(1):E4.

4. De Benedictis A, Trezza A, Carai A, et al. Robot-assisted procedures in pediatric neurosurgery. Neurosurg Focus. 2017;42(5):E7.

5. Dellaretti M, Reyns N, Touzet G, et al. Stereotactic biopsy for brainstem tumors: comparison of transcerebellar with transfrontal approach. Stereotact Funct Neurosurg. 2012;90(2): 79-83.

6. Lefranc M, Capel C, Pruvot-Occean AS, et al. Frameless robotic stereotactic biopsies: a consecutive series of 100 cases. J Neurosurg. 2015;122(2):342-352.

7. Minchev G, Kronreif G, Ptacek W, et al. A novel robot-guided minimally invasive technique for brain tumor biopsies. $J$ Neurosurg. 2020;132(1):150-158.

8. Quick-Weller J, Lescher S, Kashefiolasl S, et al. Benefit of stereotactic procedures in a series of 43 children. J Child Neurol. 2016;31(7):907-912.

9. Terrier L, Gilard V, Marguet F, et al. Stereotactic brain biopsy: evaluation of robot-assisted procedure in 60 patients. Acta Neurochir (Wien). 2019;161(3):545-552.

10. Yasin H, Hoff HJ, Blümcke I, Simon M. Experience with 102 frameless stereotactic biopsies using the neuromate robotic device. World Neurosurg. 2019;123:e450-e456.

11. Carai A, Mastronuzzi A, De Benedictis A, et al. Robot-assisted stereotactic biopsy of diffuse intrinsic pontine glioma: a single-center experience. World Neurosurg. 2017;101:584-588.

12. Coca HA, Cebula H, Benmekhbi M, et al. Diffuse intrinsic pontine gliomas in children: interest of robotic frameless assisted biopsy. A technical note. Neurochirurgie. 2016;62(6): 327-331.

13. Dawes W, Marcus HJ, Tisdall M, Aquilina K. Robot-assisted stereotactic brainstem biopsy in children: prospective cohort study. J Robot Surg. 2019;13(4):575-579.

14. Haegelen C, Touzet G, Reyns N, et al. Stereotactic robotguided biopsies of brain stem lesions: experience with 15 cases. Neurochirurgie. 2010;56(5):363-367.

15. Hoshide R, Calayag M, Meltzer H, et al. Robot-assisted endoscopic third ventriculostomy: institutional experience in 9 patients. J Neurosurg Pediatr. 2017;20(2):125-133.

16. Kwoh YS, Hou J, Jonckheere EA, Hayati S. A robot with improved absolute positioning accuracy for CT guided stereotactic brain surgery. IEEE Trans Biomed Eng. 1988;35(2): 153-160.

17. Drake JM, Joy M, Goldenberg A, Kreindler D. Computerand robot-assisted resection of thalamic astrocytomas in children. Neurosurgery. 1991;29(1):27-33.

18. Hamisch C, Kickingereder P, Fischer M, et al. Update on the diagnostic value and safety of stereotactic biopsy for pediatric brainstem tumors: a systematic review and meta-analysis of 735 cases. J Neurosurg Pediatr. 2017;20(3):261-268.

19. Kickingereder P, Willeit P, Simon T, Ruge MI. Diagnostic value and safety of stereotactic biopsy for brainstem tumors: a systematic review and meta-analysis of 1480 cases. Neurosurgery. 2013;72(6):873-882. 
20. Dhawan S, He Y, Bartek J Jr, et al. Comparison of framebased versus frameless intracranial stereotactic biopsy: systematic review and meta-analysis. World Neurosurg. 2019; 127:607-616.e4, e604.

21. Woodworth GF, McGirt MJ, Samdani A, et al. Frameless image-guided stereotactic brain biopsy procedure: diagnostic yield, surgical morbidity, and comparison with the framebased technique. J Neurosurg. 2006;104(2):233-237.

22. Louis DN, Perry A, Reifenberger G, et al. The 2016 World Health Organization Classification of Tumors of the Central Nervous System: a summary. Acta Neuropathol. 2016;131(6): 803-820.

23. Tsai EC, Santoreneos S, Rutka JT. Tumors of the skull base in children: review of tumor types and management strategies. Neurosurg Focus. 2002;12(5):e1.

24. Freeman CR, Farmer JP. Pediatric brain stem gliomas: a review. Int J Radiat Oncol Biol Phys. 1998;40(2):265-271.

25. Cooney T, Lane A, Bartels U, et al. Contemporary survival endpoints: an International Diffuse Intrinsic Pontine Glioma Registry study. Neuro Oncol. 2017;19(9):1279-1280.

26. Albright AL, Packer RJ, Zimmerman R, et al. Magnetic resonance scans should replace biopsies for the diagnosis of diffuse brain stem gliomas: a report from the Children's Cancer Group. Neurosurgery. 1993;33(6):1026-1030.

27. Khuong-Quang DA, Buczkowicz P, Rakopoulos P, et al. K27M mutation in histone H3.3 defines clinically and biologically distinct subgroups of pediatric diffuse intrinsic pontine gliomas. Acta Neuropathol. 2012;124(3):439-447.

28. Gupta N, Goumnerova LC, Manley P, et al. Prospective feasibility and safety assessment of surgical biopsy for patients with newly diagnosed diffuse intrinsic pontine glioma. Neuro Oncol. 2018;20(11):1547-1555.

29. Mueller S, Jain P, Liang WS, et al. A pilot precision medicine trial for children with diffuse intrinsic pontine gliomaPNOC003: a report from the Pacific Pediatric Neuro-Oncology Consortium. Int J Cancer. 2019;145(7):1889-1901.

30. Souweidane MM, Kramer K, Pandit-Taskar N, et al. Convection-enhanced delivery for diffuse intrinsic pontine glioma: a single-centre, dose-escalation, phase 1 trial. Lancet Oncol. 2018;19(8):1040-1050.

31. Panditharatna E, Kilburn LB, Aboian MS, et al. Clinically relevant and minimally invasive tumor surveillance of pediatric diffuse midline gliomas using patient-derived liquid biopsy. Clin Cancer Res. 2018;24(23):5850-5859.
32. Auner GW, Koya SK, Huang C, et al. Applications of Raman spectroscopy in cancer diagnosis. Cancer Metastasis Rev. 2018;37(4):691-717.

\section{Disclosures}

The authors report no conflict of interest concerning the materials or methods used in this study or the findings specified in this paper.

\section{Author Contributions}

Conception and design: all authors. Acquisition of data: Gupta, Santiago-Dieppa, Crawford, Levy, Gonda. Analysis and interpretation of data: all authors. Drafting the article: all authors. Critically revising the article: all authors. Reviewed submitted version of manuscript: all authors. Approved the final version of the manuscript on behalf of all authors: Gupta. Statistical analysis: Gupta, Yekula. Administrative/technical/material support: Gupta, Crawford, Levy, Gonda. Study supervision: Crawford, Levy, Gonda.

\section{Supplemental Information}

Online-Only Content

Supplemental material is available with the online version of the article.

Supplemental Figure and Tables. https://thejns.org/doi/suppl/ 10.3171/2020.7.PEDS20373.

\section{Previous Presentations}

Portions of this work were presented in abstract form as a poster at the American Academy of Pediatrics meeting, November 2-6, 2018, in Orlando, Florida.

\section{Correspondence}

Mihir Gupta: University of California, San Diego, La Jolla, CA. mig044@health.ucsd.edu. 